\title{
Resolving the nature of compact ultra-steep spectrum sources
}

\author{
Megan Argo*; Rachel Steadman \\ JBCA, University of Manchester, Oxford Road, Manchester, M13 9PL, UK \\ E-mail: megan.argo@gmail.com
}

\section{Zsolt Paragi}

Joint Institute for VLBI in Europe, Postbus 2, 7990 AA Dwingeloo, The Netherlands

E-mail: zparagiejive.nl

\section{Hans-Rainer Klöckner}

Subdepartment of Astrophysics, University of Oxford, Denys-Wilkinson Building, Keble Road, Oxford OX1 3RH, UK, Max-Planck-Institut für Radioastronomie, Auf dem Hügel 69, D-53121

Bonn, Germany

E-mail: hrkeastro.ox.ac.uk

\section{Huub Röttgering}

Leiden Observatory, Leiden University, Niels Bohrweg 2, NL-2333 CA Leiden, The Netherlands

E-mail: rottgeringestrw. leidenuniv.nl

\begin{abstract}
We describe ongoing efforts to investigate a sample of ultra-steep spectrum radio sources. The sources in our sample are compact on arcsecond scales, counterparts in other wavebands are rare, and their origin is, as yet, unknown. Suggestions for their nature include high-redshift radio galaxies located at or near the epoch of re-ionisation, Galactic pulsars, young obscured radio galaxies, and quasars with steep spectrum cores. In this contribution we show new 1.4-GHz eMERLIN maps of several sources from the sample, and briefly describe our ongoing observational programme.
\end{abstract}

12th European VLBI Network Symposium and Users Meeting

7-10 October 2014

Cagliari, Italy

* Speaker.

$\dagger$ e-MERLIN is a National Facility operated by the University of Manchester at Jodrell Bank Observatory on behalf of STFC. The research leading to these results has received funding from the European Commission Seventh Framework Programme (FP/2007-2013) under grant agreement No 283393 (RadioNet3)

$¥$ Summer student at JBCA, supported by the Nuffield Foundation. 


\section{A small population of puzzling sources}

A recent VLBI study of a small sub-sample of sources with ultra steep radio spectra [1] has shown that a significant fraction are compact on mas scales. At high resolution less than $30 \%$ of the flux is recovered, suggesting source structure on intermediate scales to which VLBI is insensitive. While the sample size in the previous study was too small to draw statistical conclusions (only four were observed), the full sample consists of 73 sources selected from four large catalogues (VLSS, WENSS, NVSS, FIRST). These sources are widely distributed across the survey overlap area (Figure 1), are compact on arcsecond scales, and have low-frequency spectral indices steeper than -1.2 between 74 and $330 \mathrm{MHz}$. The sample selection is described in more detail in our previ-

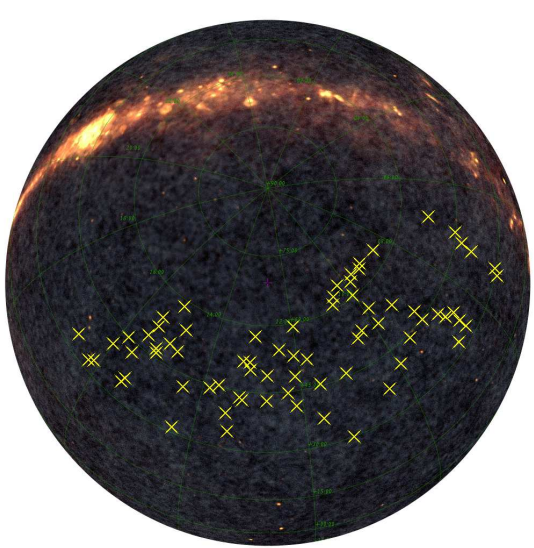

Figure 1: An all-sky plot from Aladin, using the Planck map as a background, illustrating the sky distribution of our sample. ous study [1].

Our current observational efforts are designed to examine the morphologies of these sources on angular scales intermediate between those of FIRST and VLBI, investigate whether their spectral indices stay steep above $1.4 \mathrm{GHz}$, and create a sample of sufficiently compact sources for further VLBI follow-up. Many more such sources are likely to be discovered in surveys planned or already under-way with telescopes such as LOFAR and the MWA, and this new information will allow us to more thoroughly investigate this class of enigmatic sources and narrow down the possibilities for their origin.

\section{Recent observations}

An initial survey of just five sources with the e-EVN showed that there is variety in the source properties at resolutions of a few milliarcseconds [1]. A further survey of 50 sources from the sample was carried out at $1.4 \mathrm{GHz}$ with e-MERLIN during 2013/4; these observations were made in "snapshot" mode. For e-MERLIN, with only six telescopes, the $u v$-coverage is poor for short integrations, even with the much wider bandwidths of the new receivers installed as part of the recent upgrades. Because of this limitation, these observations were scheduled in batches where the array cycled around several sources (and their associated phase calibrators) over a run of several hours. Using this technique, each source was observed for a total of one hour, but in short scans spread out in hour angle to improve the $u v$-coverage and allow reasonable images to be produced. Some results of these observations are shown in Figure 2. Of the sources observed so far, we have detected roughly one third. Most are compact, but some are clearly extended on e-MERLIN scales.

\section{Next steps}

The new e-MERLIN observations described here have given us a better idea of what fraction 

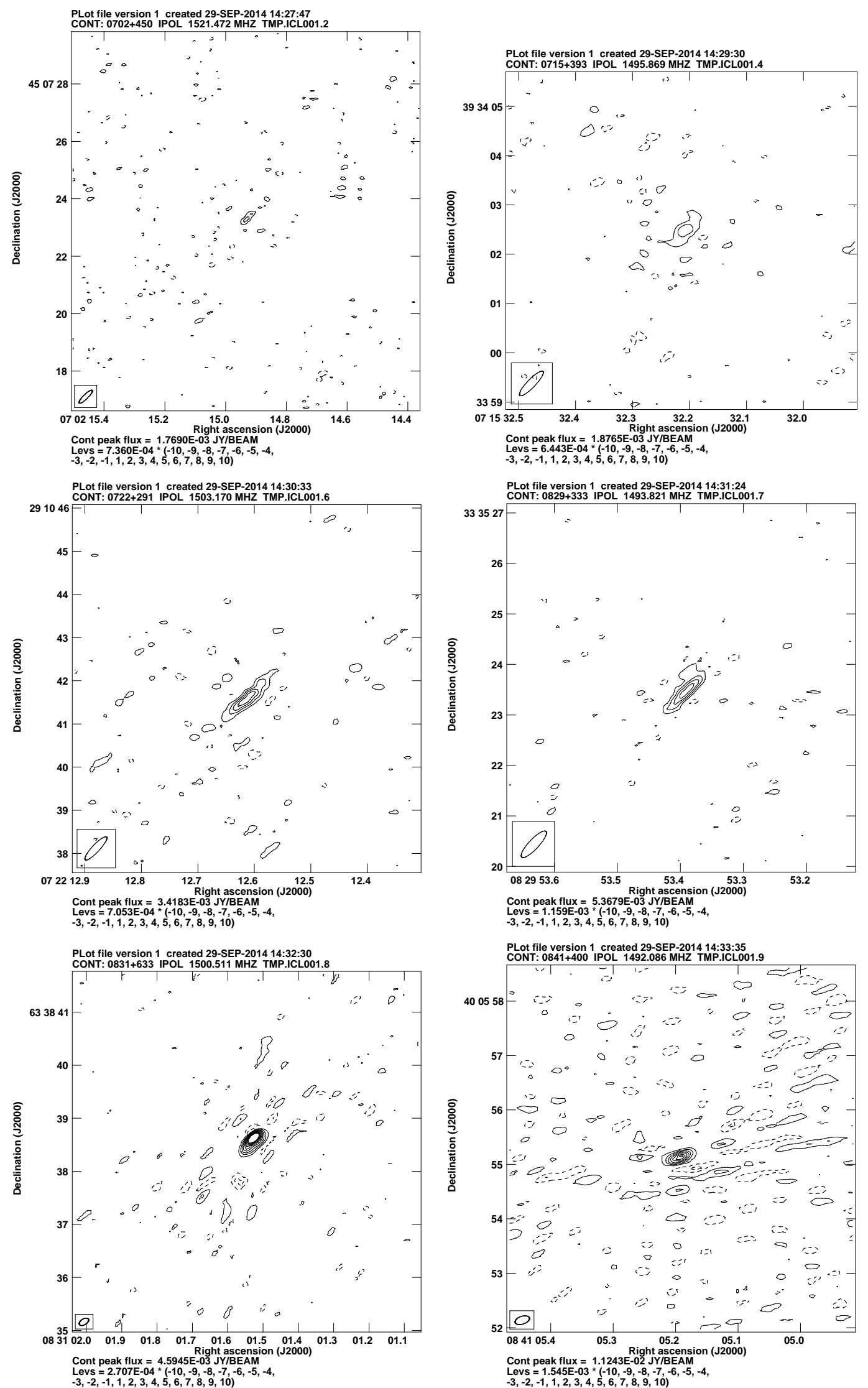

Figure 2: A sub-sample of the e-MERLIN detections made at $1.4 \mathrm{GHz}$. Of the sources observed in this programme so far, roughly one third have been detected. 

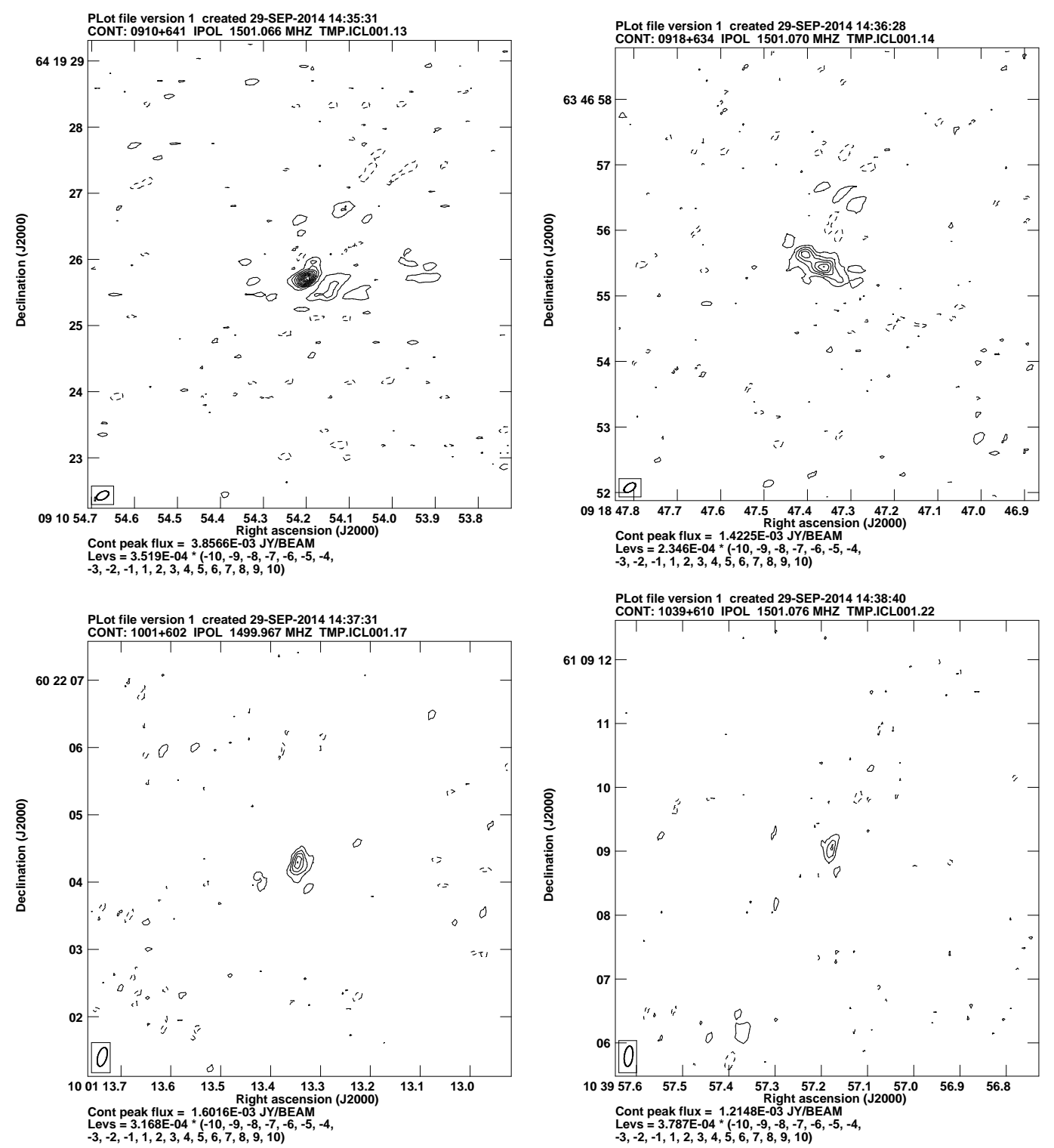

Figure 2: continued.

of these sources are compact on 100-mas scales, and of how much flux is missing between FIRST and e-MERLIN scales, despite the images having poor image fidelity due to the poor $u v$-coverage. We now have time with the JVLA to observe the entire sample in snapshot mode at C-band in order to see whether the spectral indices remain steep at higher frequencies. Further observations are also planned with e-MERLIN, before further follow-up with the EVN to examine the morphology on milliarcsecond scales.

\section{References}

[1] M.K. Argo et al., Probing the nature of compact ultra-steep spectrum radio sources with the e-EVN and e-MERLIN, MNRAS Letters 431 L58 (2013) [astro-ph/1301 . 4813] 